\title{
Flexible all-PM NALM Yb:fiber laser design for low-noise frequency comb applications and single-cavity dual-comb generation
}

\author{
Oliver H. Heckl \\ Christian Doppler Laboratory for Mid-IR Spectroscopy and Semiconductor Optics, Faculty Center for Nano Structure Research, Faculty of \\ Physics, University of Vienna, Boltzmanngasse 5, 1090 Vienna, Austria
}

Dual-comb systems traditionally consist of two mode-locked lasers actively stabilized to each other, leading to expensive and complex systems [1]. A very promising simplification is the single-cavity approach, where two pulse trains are generated within a single laser cavity [2]. Here, we present dual-comb generation from a single all-polarization-maintaining Ytterbium (Yb)-fiber laser via spectral subdivision. The laser is mode-locked using a nonlinear amplifying loop mirror (NALM) with a non-reciprocal phase bias. This versatile architecture allows for both single- and dual-comb operation in various intracavity group delay dispersion regimes.

In the single-comb operation mode, we have characterized the intensity noise, timing jitter and the free-running linewidth of the carrier-envelope-offset (CEO) frequency of five representative mode-locking states ranging from anomalous $\left(-0.035 \mathrm{ps}^{2}\right)$ to normal $\left(+0.015 \mathrm{ps}^{2}\right)$ intracavity dispersion [3]. We show that operation points far from the spontaneous emission peak of $\mathrm{Yb}(\sim 1030 \mathrm{~nm})$, but close to zero intracavity dispersion can be found, where the influence of pump noise is suppressed so strongly that the CEO-linewidth can be reduced to the single-digit $\mathrm{kHz}$ level at $1 \mathrm{~s}$ integration time without any active stabilization.

Dual-color operation in such a laser is achieved by adding a thin blade-shaped beam block in the grating compressor where the light is spatially dispersed, see Fig. 1(a). The laser emits two pulse trains centered around $1030 \mathrm{~nm}$ and $1060 \mathrm{~nm}$, respectively. By tuning the cavity dispersion, we can vary the difference in repetition rates from $\sim 1 \mathrm{kHz}$ to $10 \mathrm{kHz}$ around a nominal repetition rate of $77 \mathrm{MHz}$, which corresponds to a maximum non-aliasing bandwidth of $\sim 2.5 \mathrm{THz}$. The spectral overlap necessary for dual-comb operation is realized outside of the laser cavity by amplification and subsequent nonlinear broadening. The usability of this dual-comb setup is demonstrated by measuring the transmission of a Zinc Selenide (ZnSe) etalon.

The single-cavity dual-color/dual-comb all-PM Yb:fiber laser platform [4] presented here can easily be combined with power scaling stages and subsequent nonlinear wavelength conversion to expand its spectral operation range. Hence, such lasers show great potential as a versatile tool for many applications in spectroscopy and beyond.
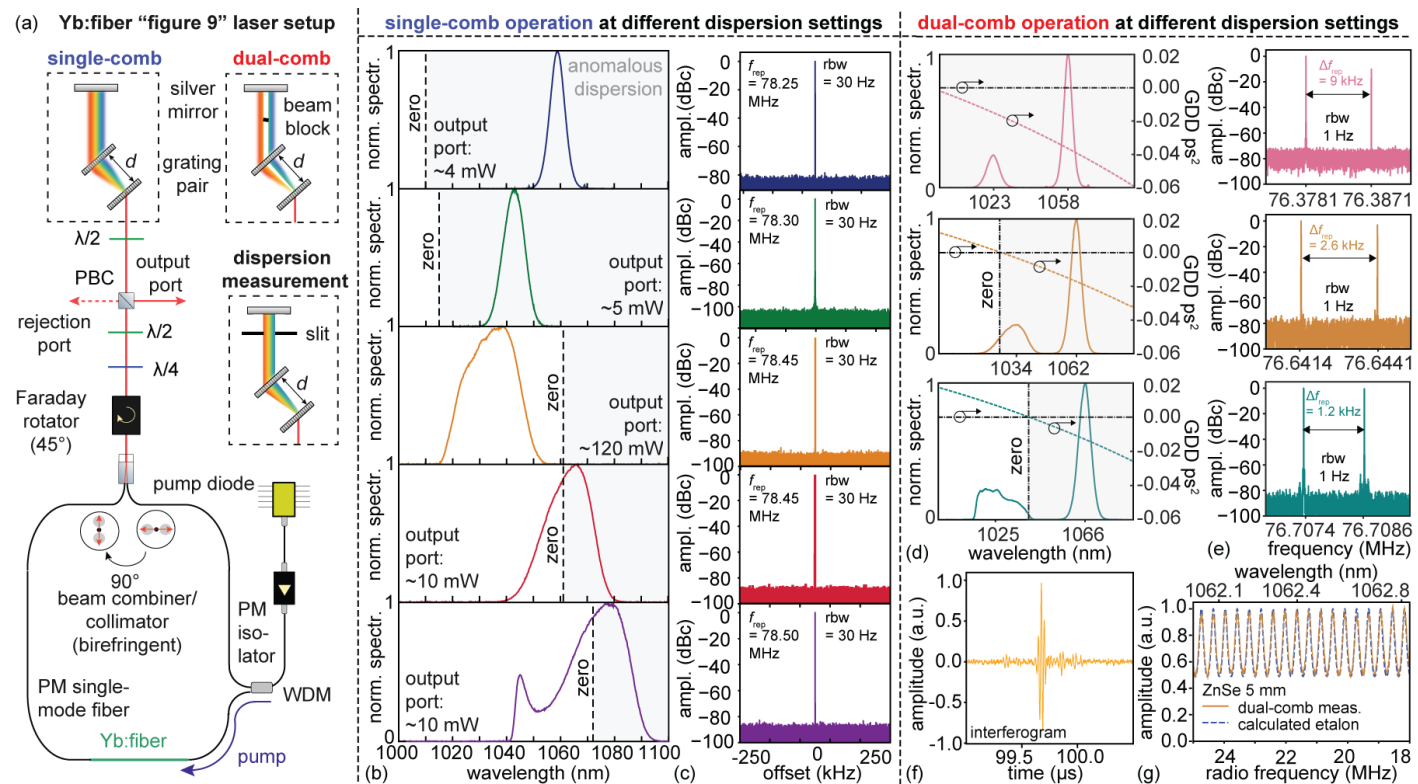

Figure 1: (a) Laser setup depicting the single- and dual-comb operation mode as well as the slit-method used for intra-cavity dispersion measurements. (b) Optical spectra of modelocking states obtained in single-comb operation at 5 different grating separations $d$ and (c) corresponding measurement of the repetition rate frequency $f_{\text {rep. }}$ (d) Optical spectra in dual-comb operation at 3 different grating separations and (e) corresponding measurement of the repetition rate frequencies $f_{\text {rep }, 1}$ an $f_{\text {rep }, 2}$. (f) Single-shot time domain interferogram of the configuration with $\Delta f_{\text {rep }}=2.6 \mathrm{kHz}$ corresponding to $(\mathrm{g})$ transmission measurement of a 5-mm thick $\mathrm{ZnSe}$ (orange) and simulation (blue)

\section{References:}

[1] I. Coddington, N. Newbury, and W. Swann, "Dual-comb spectroscopy," Optica, vol. 3, no. 4, p. 414, 2016.

[2] T. Ideguchi, T. Nakamura, Y. Kobayashi, and K. Goda, "Kerr-lens mode-locked bidirectional dual-comb ring laser for broadband dual-comb spectroscopy," Optica, vol. 3, no. 7, p. 748, 2016.

[3] A. S. Mayer et al., "Flexible all-PM NALM Yb:fiber laser design for frequency comb applications: operation regimes and their noise properties," Opt. Express, vol. 28, no. 13, pp. 18946-18968, 2020.

[4] J. Fellinger et al., "Tunable dual-comb from an all-polarization-maintaining single-cavity dual-color Yb:fiber laser," Opt. Express, vol. 27 , no. 20 , p. 28062 , Jun. 2019 